\title{
THE NATIONAL CENTER FOR CRYOEM ACCESS AND TRAINING : NATIONWIDE ACCESS TO CRYOEM TECHNOLOGY AND CURRICULA
}

Edward Eng, Eugene Chua, Mahira Aragon, Elina Kopylov, Cathleen Castello, Charlie Dubbeldam, Clint Potter and Bridget Carragher

New York Structural Biology Center, New York, New York, United States

Cryogenic electron microscopy (cryoEM) is an emerging technology that aids the study of biological systems that are not accessible to other structural methods. Recent technical advances have made cryoEM capable of generating reliable atomic models of complete and fully functional macromolecular complexes to provide a greater understanding of biological function. This technology has also enabled high-resolution analysis of previously inaccessible levels of biological organization, ranging from below 100kD to whole cells. The NIH CommonFund established the Transformative High-Resolution Cryo-Electron Microscopy program to create national cryoEM service centers to provide access to the technology and support the development of cryoEM training curricula to build a skilled workforce. In support of this, the NIH sponsored centers have created a joint website to aid in disseminating cryoEM resources from the network [1]. The National Center for CryoEM Access and Training (NCCAT) service center [2] based in New York provides scientists with access to state-of-the-art cryoEM technology and cross-training, from sample preparation [3,4] to collection of high-resolution data and computational analysis [5,6]. A key component of the NCCAT access categories is the embedded cross-training program to train a scientist to become an independent cryoEM researcher. During this intensive immersion program, a trainee "embeds" by spending significant time at NCCAT whether on-site or remote. A custom syllabus for each trainee is designed to encompass a full range of expectations, from the basics required to ensure that users produce valid structures, through more detailed training for users seeking independence. This cross-training platform is based on modular curricula that enable laboratories from structural biology and other fields to learn all steps of this technology: the preparation and evaluation of their samples for cryoEM, collection of high-resolution data, theory, and computational analysis. To support adoption in laboratories with limited exposure to structural biology, our modules include not only hands-on instrumentation instruction but the availability of distance learning materials on cryoEM methodology. At the center, we bring the most current best practices to assist researchers to access new technologies and accelerate their research. Our driving aim is to increase the number of investigators who can independently practice cryoEM to enable researchers in other fields of structural biology to adopt cryoEM methods. Our cross-training efforts are intended to serve as a catalytic effect by increasing research capacity by helping investigators to work independently, rather than by solving their structures for them. Taken together we aim to lower the barriers of access and cross-train biomedical researchers to broadly utilize cryoEM techniques.

\section{References}

[1] https://cryoemcenters.org

[2] https://nccat.nysbc.org

[3] Razinkov et al. A new method for vitrifying samples for cryo-EM. J Struct Biol. 2016;195(2):190-8.

[4] Dandey et al. Spotiton: New features and applications. J Struct Biol. 2018; 202(2):161-169.

[5] Cheng et al, Leginon: New features and applications Protein Sci. 2021; 30(1):136-150.

[6] Lander et al, J Struct Biol. 2009; 166(1):95-102.

[7] Some of this work was performed at the National Center for Cryo-EM Access and Training (NCCAT) and the Simons Electron Microscopy Center located at the New York Structural Biology Center, supported by the NIH Common Fund Transformative High Resolution Cryo-Electron Microscopy program (U24 GM129539,) and by grants from the Simons Foundation (SF349247) and NY State Assembly. 